Cite this: RSC Adv., 2014, 4, 19380

\title{
Influence of the relative humidity on the morphology of inkjet printed spots of IgG on a non-porous substrate
}

\author{
Liyakat Hamid Mujawar, $\uparrow^{\mathrm{ab}}$ J. G. M. Kuerten, ${ }^{\mathrm{cd}}$ D. P. Siregar, ${ }^{\mathrm{c}}$ Aart van Amerongen ${ }^{\star a}$ \\ and Willem Norde be
}

During the drying of inkjet printed droplets, the solute particles (IgG-Alexa-635 molecules) in the drop may distribute unevenly on the substrate, resulting in a "coffee-stain" spot morphology. In our study, we investigated the influence of the relative humidity on the distribution of inkjet printed fluorophore labeled IgG molecules on a polystyrene substrate. A theoretical model for an evaporating droplet was developed in order to predict the changes in the spot diameter, height and volume of a drying droplet. An experiment was performed where a sessile droplet was monitored using a CCD camera installed on a goniometer and good agreement was found between the experimental results and simulation data. We also compared the predicted morphology for an inkjet-printed microarray spot with the experimental results where IgG molecules were printed for various relative humidities. The spot morphology of the dried spots was analyzed by a confocal laser microscopy. At a lower relative humidity (i.e., $<60 \%$ ), a spot morphology resembling a coffee stain was prominent, whereas a more homogeneous distribution was observed when droplets were printed and dried at a higher relative humidity ( $70 \%)$.

Received 14th February 2014 Accepted 28th February 2014

DOI: $10.1039 / c 4 r a 01327 a$

www.rsc.org/advances humidity $^{17-19}(\mathrm{RH})$ and solvent ${ }^{15,20}$ ( $\mathrm{pH}$ and composition). The most commonly observed non-homogeneous distribution of inkjet printed spots, often termed the 'coffee-stain' effect, was studied in detail by Deegan et al. ${ }^{21-23}$ Deegan mentioned three conditions for the coffee-stain shape: pinning of the contact line, a higher evaporation rate at the edge of the droplet and a volatile solvent. Since surface tension tends to keep the drop in the shape of a spherical cap during the evaporation of the solvent, a pinned contact line and a higher evaporation rate at the edge of the droplet result in a flow of the solution toward the edge of the drop, thereby causing the coffee-stain effect. Sommer and Rozlosnik ${ }^{\mathbf{2}}$ argued that the coffee-stain effect can also take place for an unpinned contact line. An extension to this model was proposed by Fischer, ${ }^{25}$ where the shape of the droplet is not assumed to be spherical during the evaporation process. Van Dam and Kuerten ${ }^{\mathbf{2 6}}$ proposed an extension for the calculation of the curvature of the droplet shape in order to incorporate a less flat micro-scale droplet than was assumed in the previous model.

While printing, controlled evaporation of the droplet ${ }^{17}$ (by maintaining a constant relative humidity) is of crucial importance. In our research, we have demonstrated the influence of various relative humidities $(40 \pm 1 \% / 50 \pm 1 \% / 60 \pm 1 \%$ / $70 \pm 1 \%$ and $80 \pm 1 \%$ ) on the distribution of inkjet printed biomolecules. Using a non-contact microarrayer, fluorophorelabeled antibody molecules (IgG-Alexa-635) were printed and dried under similar conditions and the spot morphology of the biomolecules in the dried spots was analyzed by confocal laser 
scanning microscopy. From the observed profiles of the spots, we could determine the distribution pattern of the IgG molecules printed and dried at various relative humidities.

Furthermore, we present a mathematical model for the fluid dynamics and the distribution of the solute molecules. The model is based on considering three major aspects: the flow of the liquid due to evaporation, convection and diffusion of the solute, and binding of the solute molecules to the substrate.

\section{Materials and methods}

\subsection{Mathematical model}

The mathematical model covers the dynamics of the solvent due to evaporation, the change in concentration of the solute and the binding of the solute molecules to the surface. Fig. 1 depicts an axially symmetric droplet on a smooth horizontal substrate, where $h$ denotes the height of the droplet, and $z$ and $r$ are the vertical and radial coordinates.

A complete model for the flow inside the droplet is provided by the three-dimensional Navier-Stokes equation and the continuity equation for an incompressible fluid. However, a study of the order of the magnitude of the terms in these equations reveals that the model can be simplified by the lubrication approximation. ${ }^{25-28}$ The most important assumption in this simplified model is that the Reynolds number is so small that the convective terms in the Navier-Stokes equation are negligible. This assumption leads to a simplified form of the Navier-Stokes equation in case the height of the droplet is small compared to its radius. In this approach, the radial velocity component of the solvent, $u$, can explicitly be determined from the shape of the droplet and the pressure difference between the inside and the outside of the droplet at the liquid-air interface: ${ }^{28}$

$$
u=\frac{2}{\mu}\left(\frac{1}{2} z^{2}-h z\right) \frac{1}{\mu} \frac{\partial p}{\partial r}
$$

Here, $\mu$ is the dynamic viscosity of the solution and $p$ the pressure at the interface between the drop and the surrounding air. The shape of the droplet, $h(r)$, is determined by conservation of mass, which incorporates changes in shape due to the flow inside the droplet and due to evaporation ${ }^{28}$

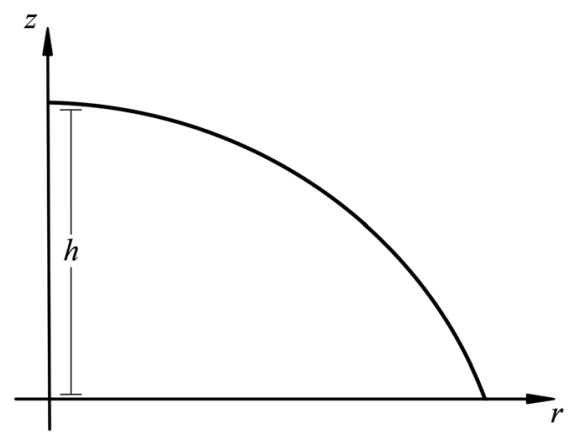

Fig. 1 Schematic representation of a spherical droplet on an impermeable substrate.

$$
\frac{\partial h}{\partial t}=\frac{1}{3 \mu} \frac{1}{r} \frac{\partial}{\partial r}\left(r h^{3} \frac{\partial p}{\partial r}\right)-J(r)
$$

where $t$ is the time and $J(r)$ indicates the evaporation velocity, which may depend on the radial coordinate. Evaporation of the droplet is induced by a normal gradient of vapor pressure at the droplet-liquid interface. The vapor pressure gradient depends on the relative humidity in the ambient air and on the temperature-dependent saturation pressure. An evaporation model by Popov ${ }^{29}$ and Siregar $e t a l .{ }^{30}$ is applied to describe the mass transfer over the liquid-air interface.

The pressure within the droplet is determined by the surface tension and the local curvature of the droplet-air interface, according to the Laplace pressure ${ }^{30}$

$$
p=-\sigma \frac{1}{r} \frac{\partial}{\partial r}\left(\frac{r}{\sqrt{1+\left(\frac{\partial h}{\partial r}\right)^{2}}} \frac{\partial h}{\partial r}\right)
$$

The term in the denominator accounts for the exact radius of the curvature of an axially symmetric drop. It allows us to extend the range of the validity of the lubrication approximation to the case with a larger ratio between the height and radius. In case the contact line of the droplet is not pinned to the substrate, the dynamics of the contact line are incorporated in the model by adding the disjoining pressure to (3). ${ }^{30}$ The disjoining pressure, which accounts for the molecular interaction near the contact line, is only unequal to zero in a small region near the contact line and keeps the contact angle constant.

We will also apply the lubrication approximation to cases where the height and radius of the droplet are almost equal. For the calculation of the shape history of the droplet during evaporation, the lubrication approximation has no influence, since the surface tension keeps it in a spherical cap shape. During the first part of the evaporation, when the contact angle is still large, the velocity profile in the droplet is not exactly given by the solution of the lubrication approximation, but it turns out that the deviations are small and have a very small effect on the solute concentration.

During the evaporation process, the change in the solute concentration is determined by three physical phenomena: the loss of solvent by evaporation, convection and diffusion of the solute in the solvent, and the adsorption of solute molecules to the substrate. The loss of solvent not only increases the solute concentration but also leads to transport of the solute by diffusion. Adsorption leads to a local decrease of the solute concentration in the region near the liquid-substrate interface. Hence, the concentration distribution of the solute is governed by a two dimensional convection-diffusion equation ${ }^{30}$

$$
\frac{\partial C}{\partial t}=-\frac{1}{r} \frac{\partial}{\partial r}(r C u)-\frac{\partial}{\partial z}(C w)+D \frac{1}{r} \frac{\partial}{\partial r}\left(r \frac{\partial C}{\partial r}\right)+D \frac{\partial^{2} C}{\partial z^{2}}-F \delta(z)
$$

where $C$ is the solute concentration in the droplet, $u$ and $w$ are radial and axial velocity components, respectively, and $D$ is the 
diffusivity of the solute particles. The function $F$ describes the mass loss of the solute in mass per unit area due to the binding between the molecules and surface.

The adsorption of the solute determines the surface coverage and influences the function of the biomolecule. Here, we make use of a model by Kurrat et al. ${ }^{31}$ where adsorption may happen in a reversible and an irreversible way. This model describes the dependency of the mass adsorption rate on the concentration of the solute particles near the liquid-substrate interface, $C_{\mathrm{s}}$. We indicate the reversibly and irreversibly adsorbed mass per unit area by $M_{\mathrm{r}}$ and $M_{\mathrm{i}}$, respectively. The rates of adsorption for the reversible and irreversible processes are given by ${ }^{32}$

$$
\begin{gathered}
\frac{\partial M_{\mathrm{r}}}{\partial t}=k_{\mathrm{a}} C_{\mathrm{s}} \phi-\frac{k_{\mathrm{d}} M_{\mathrm{r}}}{\sqrt{\phi}} \\
\frac{\partial M_{\mathrm{i}}}{\partial t}=k_{\mathrm{s}} C_{\mathrm{s}} \phi
\end{gathered}
$$

where $k_{\mathrm{a}}$ and $k_{\mathrm{s}}$ are the rate constants for reversible and irreversible adsorption, respectively, and $k_{\mathrm{d}}$ is the rate constant for desorption. These constants can be determined experimentally and depend on the type of molecule, on the buffer that is used and on the properties of the substrate. The variable $\phi$ is the available fraction of the surface area. The mass loss $F$ defined in eqn (4) is equal to the sum of the rates for reversible and irreversible adsorption from eqn (5) and (6):

$$
F=k_{\mathrm{a}} C_{\mathrm{s}} \phi-\frac{k_{\mathrm{d}} M_{\mathrm{r}}}{\sqrt{\phi}}+k_{\mathrm{s}} C_{\mathrm{s}} \phi
$$

\subsection{Experimental}

2.2.1 Substrate and reagents. For the experimental studies, a HTA $^{\mathrm{TM}}$ polystyrene (PS) slide was used which was purchased from Greiner BioOne. For printing onto the polystyrene substrate, a $100 \mathrm{mM}$ carbonate buffer (CB) pH 9.6 was prepared in Milli-Q water with a resistivity of $18.2 \mathrm{M} \Omega \mathrm{cm}^{-1}$.

2.2.2 Biomolecules. Microarrays were produced on $\mathrm{HTA}^{\mathrm{TM}}$ polystyrene slides by printing IgG-Alexa-635, which was purchased from Invitrogen (Oregon, USA). The stock was diluted to $200 \mu \mathrm{g} \mathrm{mL}{ }^{-1}$ in $0.1 \mathrm{M} \mathrm{CB}$ (pH 9.6) and loaded into the wells of a Genetix microtiter plate (Genetix X7020, Berkshire, United Kingdom).

2.2.3 Printing of IgG. IgG-Alexa- 635 molecules were printed on the HTA $^{\mathrm{TM}}$ polystyrene slides with a non-contact spotter, sciFLEXARRAYER S3 (Scienion AG, Berlin, Germany). The printing was performed at constant temperature and humidity. The voltage and pulse of the piezo dispensing capillary (PDC) were optimized to print a droplet of $\sim 250 \mathrm{pL}$. The temperature was maintained at $\sim 21{ }^{\circ} \mathrm{C}$. On these HTA $^{\mathrm{TM}}$ slides, IgG-Alexa635 was printed at different relative humidities, i.e. $40 \pm 1 \%$, $50 \pm 1 \%, 60 \pm 1 \%, 70 \pm 1 \%$ and $80 \pm 1 \%$. The humidity within the hood was controlled and kept constant by a built-in sensor which could precisely monitor the changes in the humidity. In addition, the relative humidity inside the hood was also monitored by a thermo-hygrometer (Testo AG, Lenzkirch, Germany) with a precision of $\pm 1{ }^{\circ} \mathrm{C}$.

While printing under various relative humidities, other printing conditions, such as temperature, voltage (88 V) and pulse ( $49 \mu \mathrm{s})$, were kept constant. Prior to printing, the hood of the printer was allowed to be conditioned for 15 minutes at the set humidity value. After printing, the substrate was incubated and dried under the same conditions for one hour and stored in a sealed aluminium pouch. After overnight drying, the printed spots were analyzed by CLSM to study the spot morphology and the distribution of the IgG molecules in the spot.

\section{Instrumentation}

\subsection{Goniometer}

The influence of the relative humidity on the drying of the droplet was analyzed by monitoring the change in contact angle $(\theta)$, diameter $(D)$, volume $(V)$ and height $(h)$ of a liquid droplet using a contact angle measuring system (G10, Krüss GmbH, Hamburg, Germany). A $2 \mu \mathrm{L}$ MQ water droplet was placed on the surface of a polystyrene slide and analyzed by an in-built CCD video camera (Sony XC-77CE). All the parameters were measured with the drop analysis software (DSA-1).

The change in contact angle $(\theta)$ and volume $(V)$ for a droplet of IgG-Alexa (200 $\mu \mathrm{g} \mathrm{mL} \mathrm{mL}^{-1}$ ) was monitored at two different relative humidity levels, $\sim 19 \%$ and $\sim 75 \%$ respectively. The measurements at $19 \%$ relative humidity were performed in a room where the default humidity was $19 \pm 1 \%$. For the measurements at $75 \pm 1 \%$ relative humidity, the $\mathrm{HTA}^{\mathrm{TM}}$ PS slide was placed inside a transparent home-made chamber which was pre-saturated with water. Prior to placing the droplet on the $\mathrm{HTA}^{\mathrm{TM}}$ polystyrene surface, the relative humidity inside the chamber was monitored for one hour using a portable thermohygrometer (Testo AG, Lenzkirch, Germany). The relative humidity was found to be $75 \pm 1 \%$. This transparent chamber was positioned on the stage of the goniometer and using a micropipette, a droplet was placed on the $\mathrm{HTA}^{\mathrm{TM}}$ polystyrene surface. Two independent sets of experiments were performed to confirm the results.

\subsection{Confocal laser scanning microscopy (CLSM) imaging}

The distribution of the fluorophore-labeled IgG molecules printed at various humidities on the $\mathrm{HTA}^{\mathrm{TM}}$ PS slide was analyzed by confocal laser scanning microscopy (Carl Zeiss Axiovert 200 microscope, Zeiss, Jena, Germany), equipped with a LSM 5 Exciter. The spots were scanned at 10x magnification and the configuration of the objective was LD Plan-Neofluar 10x/0.30 Korr M27. The CLSM was set at $633 \mathrm{~nm}$ with a He-Ne laser, the size of the pinhole was $206 \mathrm{~nm}$ and the transmission was $11 \%$. The dimensions of the scanner were $X: 1272.79 \mu \mathrm{m}, Y$ : $1272.79 \mu \mathrm{m}$, respectively. The mean intensity of the spots was analyzed by "Zen 2008" software, and the homogeneity of the spots was investigated using Image J software. A cross-section profile plot for each of the nine spots was calculated and the final plot data were made after averaging these values along with the standard deviation. The total intensity for each spot was also calculated as the product of the mean intensity and the surface area of the spots. 


\subsection{Atomic force microscopy (AFM)}

The surface characteristics of the HTA-PS slide were analyzed by atomic force microscopy (Asylum MFP-3D, Santa Barbara, CA, USA) using the tapping-mode (in air). An area of $90 \mu \mathrm{m} \times 90 \mu \mathrm{m}$ was scanned (256 lines) at a frequency of $0.4 \mathrm{~Hz}$. The RMS roughness of the bare HTA-PS slide was calculated as $9.2 \mathrm{~nm}$, which implies a smooth surface relative to the drop dimensions.

\section{Results and discussion}

\subsection{Evaporation model}

The validation of the evaporation model is presented in this subsection. Numerical results are compared with experimental results from an experiment in which a droplet of MQ water was evaporated at a relative humidity of $\sim 19 \%$. The experiment has been performed twice, yielding almost equal results.

Fig. 2 shows the comparison between the numerical and experimental results during the evaporation. The experimental results clearly indicate that the contact line is not pinned: after a short initial time, the diameter monotonically decreased during the evaporation and as a result, the contact angle remains constant with time. Therefore, we included the disjoining pressure in the model with a constant contact angle of $90^{\circ}$. Fig. 2B shows the droplet volume as a function of time. During the whole evaporation process, the experimental results agree very well with the model results and show the typical behavior for an unpinned contact line, in which the rate of mass loss is proportional to the radius of the droplet. ${ }^{30}$ In Fig. 2A, it can be seen that the experimentally measured diameter is almost constant during the first stages of the evaporation process, after which it starts decreasing at the same rate as in the model. This indicates that the contact line was pinned during the first stages and then started retracting. This finding is consistent with the results observed by Bourgès-Monnier and Shanahan, ${ }^{33}$ who performed measurements on the evaporation of sessile droplets of water and $n$-decane on various substrates. They found a decreasing contact angle during the first stages of evaporation until a certain contact angle was reached. After that, the contact angle remained constant and the diameter of the droplet started decreasing. This effect was attributed to the receding contact angle. Initially, the contact angle is larger than the receding contact angle and the contact line remains pinned until the receding contact angle has been reached. Indeed, our results also show a slightly decreasing contact angle during the initial 3 minutes of the drying, after which a constant contact angle is found.

The results for the droplet height, displayed in Fig. 2C, are consistent with this explanation: initially the measured decrease in height is larger than predicted by the model, but after 2-3 minutes they decrease at the same rate. The systematic difference between the droplet height and radius in the experiment indicates that the contact angle is slightly lower than 90 degrees or that the shape is not exactly a spherical cap, which could be an effect of gravity. The results are consistent with standard models in the literature ${ }^{34,35}$ for a constant contact angle, as $V^{2 / 3}$ is a linear function of time.

\subsection{Experimental analysis of drying a liquid droplet at two different humidities ( $\sim 19 \%$ and $\sim 75 \%)$}

As shown in Section 4.1, after an initial period in which the contact angle decreased to the value of the receding contact angle,$^{33}$ an unpinned situation was observed for a pure liquid droplet drying on a substrate. We also studied the influence of two different relative humidities, i.e. $\sim 19 \%$ and $\sim 75 \%$, on the drying of a droplet of IgG-Alexa-635 $\left(200 \mu \mathrm{g} \mathrm{mL}{ }^{-1}\right)$ by monitoring the change in the contact angle $\left(\theta_{\mathrm{t}}\right)$ and volume $(V)$.

It was found that irrespective of the relative humidity used, the initial contact angle $\left(\theta_{0}\right)$ for a sessile droplet on a PS surface was $\sim 93^{\circ}$. The change in contact angle $(\theta)$ during the drying process was faster at a lower RH: in 20 seconds, $\theta$ decreased to $46^{\circ}$ at $\mathrm{RH} \sim 19 \%$, whereas at $\mathrm{RH} \sim 75 \%$, it decreased to $86^{\circ}$ (Fig. 3A). These observations are in agreement with the studies performed earlier by Lages et al., who used modified gold surfaces and observed the change in the contact angle $(\theta)$ for aqueous solutions under controlled conditions. ${ }^{36}$ Irrespective of the relative humidity used for drying, the contact angle changed with time (see Fig. 3A), thus implying that, in contrast to a droplet of MQ water, the contact line of a liquid droplet containing IgG molecules was pinned.

The influence of the relative humidity on the drop volume history was also significant. At $\mathrm{RH} \sim 19 \%$, evaporation of the initial volume $(2 \mu \mathrm{L})$ to $0.6 \mu \mathrm{L}$ occurred in less than 20 minutes, whereas at $\mathrm{RH} \sim 75 \%$, it took 120 minutes (Fig. 3B). This difference is much larger than expected based on the dependence of the evaporation rate on the relative humidity. Our observations are in line with those presented by Liu ${ }^{18}$ et al. who, at a lower humidity (47\%), observed a significant decrease in the
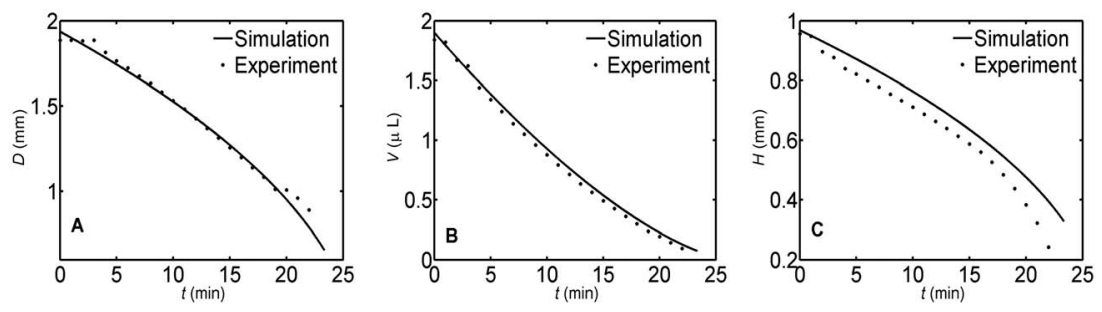

Fig. 2 Comparison between the numerical and experimental results for a MQ water droplet with an initial volume of $2 \mu L$ and relative humidity $\mathrm{RH}=0.19$. The numerical simulation is performed with the model for the droplet with an unpinned contact line on the PS surface. The comparison shows the droplet (A) diameter, (B) volume and (C) height, as functions of time. 

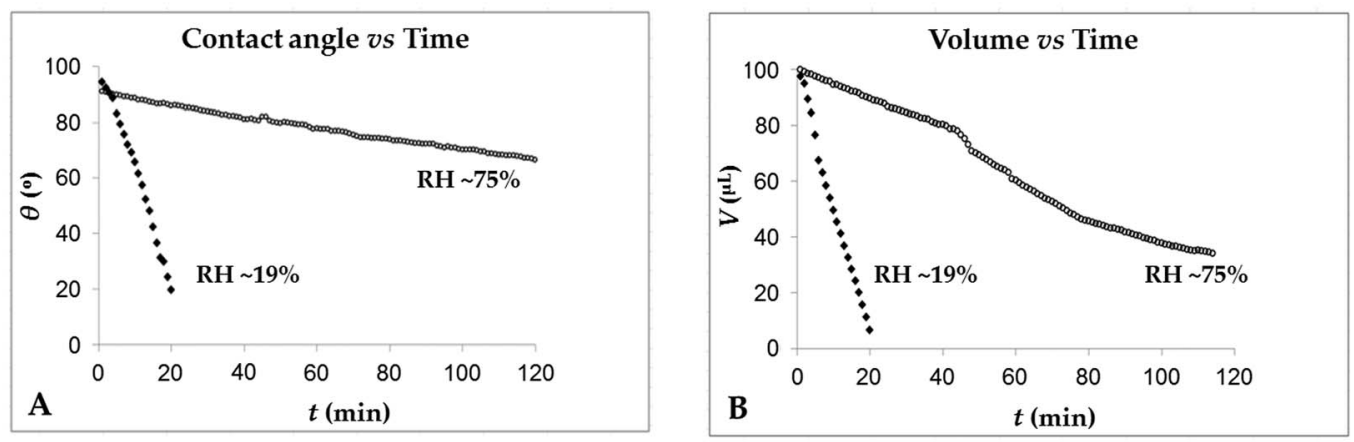

Fig. 3 The measured influence of humidity on the drying of a droplet containing IgG-Alexa-635 at two different relative humidities ( $\sim 19 \%$ and $\sim 75 \%$ ) as monitored by a goniometer; (A) change in the contact angle $(\theta)$ and (B) change in the volume.

sessile drop volume of pure water compared to the same experiment at a higher humidity (80\%).

The data presented in Section 4.1 (i.e., for pure MQ water) depicts the unpinned contact line situation whereas in the presence of IgG molecules, the contact line is pinned. This contrasting behaviour in the presence and absence of protein molecules has already been explained by Choi et al. ${ }^{37}$ According to these authors, in an evaporating droplet the protein molecules tend to adsorb on the substrate surface, thus reducing its surface hydrophobicity. With increasing protein concentration, pinning of the contact line is promoted, which, in turn, influences the contact angle of the liquid droplet.

\subsection{Influence of the relative humidity on mass distribution}

In this subsection, the influence of the relative humidity on the deposited solute mass is described. The relative humidity determines the magnitude of the evaporation term compared to the convective term in the evolution equation for the droplet height, eqn (1). In this study, we chose relative humidity values ranging between 0.3 and 0.9 and considered a droplet with a pinned contact line, an initial volume of $250 \mathrm{pL}$ and a diameter of $100 \mu \mathrm{m}$. The consideration of a pinned contact line model can be well understood with the results explained in Section 4.2. We choose higher rate constants for adsorption than Kurrat et al., ${ }^{31}$ in order to take into account the effect of the hydrophobicity ${ }^{32}$ of the substrate in our case.

According to the value for the area of a single molecule adopted by Kurrat et al., ${ }^{31}$ the maximum possible adsorbed mass density equals $3.7 \mathrm{mg} \mathrm{m}^{-2}$, whereas the initial concentration leads to an average deposited mass density of $6.4 \mathrm{mg} \mathrm{m}^{-2}$. This implies that not all the molecules present in the solution can be adsorbed. After the liquid has completely evaporated there will still be molecules lying on the substrate, but unbound. These unbound molecules will be removed in a rinsing step after drying, whereas the adsorbed molecules will remain on the substrate.

Fig. 4A shows the profiles of the total deposited mass density, including both the adsorbed and the unbound molecules, and Fig. 4B shows the profiles of the adsorbed mass density. Without diffusion and adsorption, the deposition profile is independent of the relative humidity. The time scale increases with increasing relative humidity, leading to larger evaporation times, but the resulting deposition profile after complete drying is constant. It shows a large deposition peak at the edge of the droplet, since all the solute molecules are transported to the edge during evaporation. Adding diffusion obviously broadens this peak. Since diffusion does not depend on the relative humidity, but the convection velocity is lower at a higher relative humidity, the effect of diffusion is larger at a higher relative humidity. However, also with diffusion, the coffee-stain-shaped deposit layer is still observed for all the values of relative humidity considered.

If the effect of adsorption is also added, the slower convection at a higher relative humidity gives the molecules more time to adsorb on the substrate before they reach the edge of the droplet. Therefore, the resulting adsorption profile (Fig. 4B) is more uniform. Note that at the highest relative humidity, the maximum possible adsorbed mass density is almost reached even in the center of the droplet. At a low relative humidity, both the total deposited mass density and the adsorbed mass density show the coffee-stain shape.

\subsection{Experimental analysis of the influence of relative humidity on the fluorescence of inkjet printed IgG molecules}

Spots of fluorophore-labeled IgG molecules printed and dried on a non-porous surface showed variations in the spot morphology and fluorescence intensity depending on the ambient relative humidity. The average mean intensity and total intensity of the inkjet printed spots had a maximum when the biomolecules were printed and dried at a relative humidity of $60 \%$ (see Fig. 5).

At low humidity $(\sim 0.4)$, the morphology pattern of the spots of IgG-Alexa-635 showed a non-homogeneous distribution, which resembles a coffee-stain-shape (see Fig. 6A). The intensity profile plot clearly shows higher fluorescence at the edge of the spot compared to the overall spot area, thus confirming an inhomogeneous distribution. The higher fluorescence intensity observed at the edge of the spot is due to a higher locally deposited mass of biomolecules, as observed in the results of the model of the total mass distribution (see Fig. 4A). This 

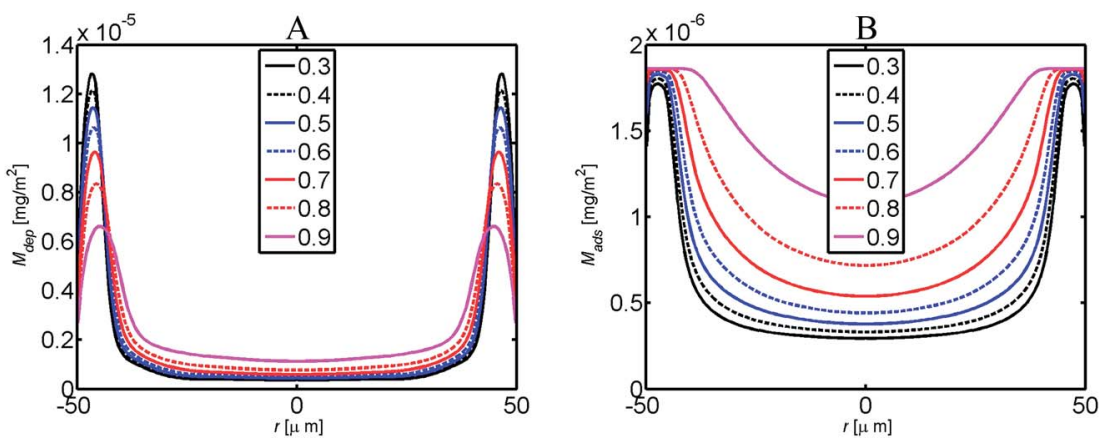

Fig. 4 Total deposited mass density (A) and adsorbed mass density (B) as functions of the radial coordinate for the various values of relative humidity simulated with the model.

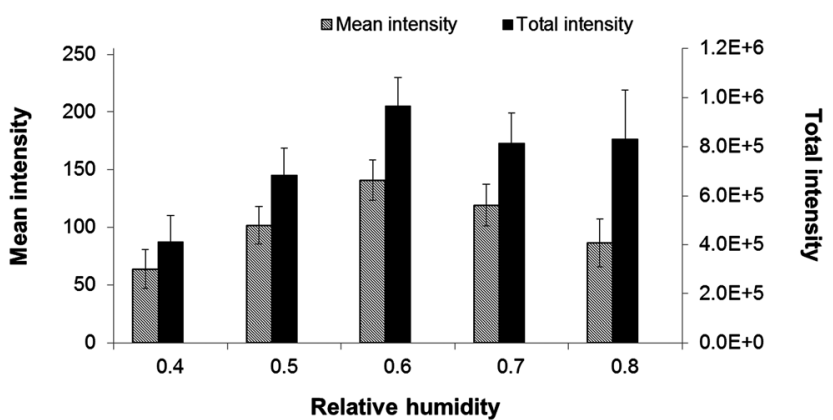

Fig. 5 Based on measured CLSM data, average mean intensity (shaded) and average total intensity (solid) of the IgG-Alexa-635 spots printed on a surface at various relative humidities.

uneven distribution results from the pinning of the contact line of the evaporating droplet, ${ }^{21}$ which in turn causes internal convection within the droplet, creating an outward flow of the IgG molecules towards the edge of the spot. ${ }^{22}$

When printing was performed at high relative humidities $(>40 \%)$, the overall uniformity of the spots increased with increasing humidity, which is consistent with the simulation results shown in the previous section. As shown in the profile diagram, the fluorescence intensity was higher at the edge of the spot when the drop was dried at $40 \pm 1 \%$ and $50 \pm 1 \%$ relative humidity, with a very low fluorescence intensity in the central part of the spot (see Fig. 6A and B). Upon raising the relative humidity to $60 \pm 1 \%$, $(\mathrm{RH} \sim 0.6)$, the drying time further increased and the coffee-stain-effect reduced compared to the drying of similar droplets at lower relative humidities (see Fig. 6C). A better and more homogeneous distribution was observed when the IgG molecules were printed at $70 \pm 1 \%$ $(\mathrm{RH} \sim 0.7)$, where the profile plot was much more uniform (see Fig. 6D). This situation may be explained by the fact that at a higher relative humidity, the droplet has a longer drying time and hence shows less internal convection. This allows the IgG molecules present within the sessile droplet to be adsorbed on the substrate rather than being transported to the edges. Moreover, at a higher relative humidity, the effect of diffusion, which counteracts high local solute concentrations, becomes more prominent compared to the effect of convection. However, although the spot morphology at $\mathrm{RH} \sim 0.7$ was more uniform than at $\mathrm{RH} \sim 0.6$, both the mean and total fluorescence intensities were higher at $\mathrm{RH} \sim 0.6$. This may indicate that the conditions for the binding of the IgG molecule at the surface may be more favorable at $\mathrm{RH} \sim 0.6$ compared to $\sim 0.7$. A further increase in the humidity (up to $\mathrm{RH} \sim 0.8$ ) resulted in increased spreading of the droplet, which was clearly demonstrated by the larger spot diameter $(117 \mu \mathrm{m})$ compared to the $96 \mu \mathrm{m}$ observed for IgG-Alexa spots printed and dried at $40 \pm 1 \%$ humidity (see Fig. 6E). Also at $\mathrm{RH} \sim 0.8$, the spot morphology was more uniform than the spot morphology at $\mathrm{RH} \sim 0.4$, although the total intensity was less than at $\mathrm{RH} \sim 0.6$ (see Fig. 6, right panel). The spots in Fig. 6 at $80 \%$ humidity are more homogeneously distributed compared to those printed at $\mathrm{RH} 40 \pm 1 \%$ or $50 \pm$ $1 \%$, and the higher fluorescence observed at the edges may be due to the formation of multi-layers during the process of evaporation. Fig. 4A shows that this excess adsorption is more likely to occur at the edges, since the number of deposited molecules is much higher there than in the central part due to convection.

A comparison of the CLSM profile plot with the total mass distribution profile also shows slightly higher mass deposition, even though the droplet was dried at $\mathrm{RH} \sim 0.8$ (compared with Fig. 4A).

The results of the numerical simulations for the case of a pinned contact line also showed similar behavior to that observed in the experiments (see Fig. 3A), i.e., when a sessile drop was allowed to evaporate at a lower relative humidity, the contact line was pinned and the resulting mass distribution was non-homogeneous, which was confirmed by experimental results as well.

Based on the plot profile diagram in Fig. 6, it can be concluded that the overall spot morphology was irregular when the IgG-Alexa-635 molecules were printed and dried at lower humidities $\left(<60^{\circ}\right)$, whereas the fluorescence intensity was distributed more evenly when higher relative humidities $\left(>60^{\circ}\right)$ were applied. The mechanism behind the non-homogeneous distribution of the molecules at lower humidity has already been demonstrated for colloidal polystyrene particles by Chhasatia ${ }^{17}$ et al. Using a CCD camera they showed that at lower humidity, the outward migration of the colloidal particles was higher, giving rise to a coffee-stain-shaped spot, whereas with 


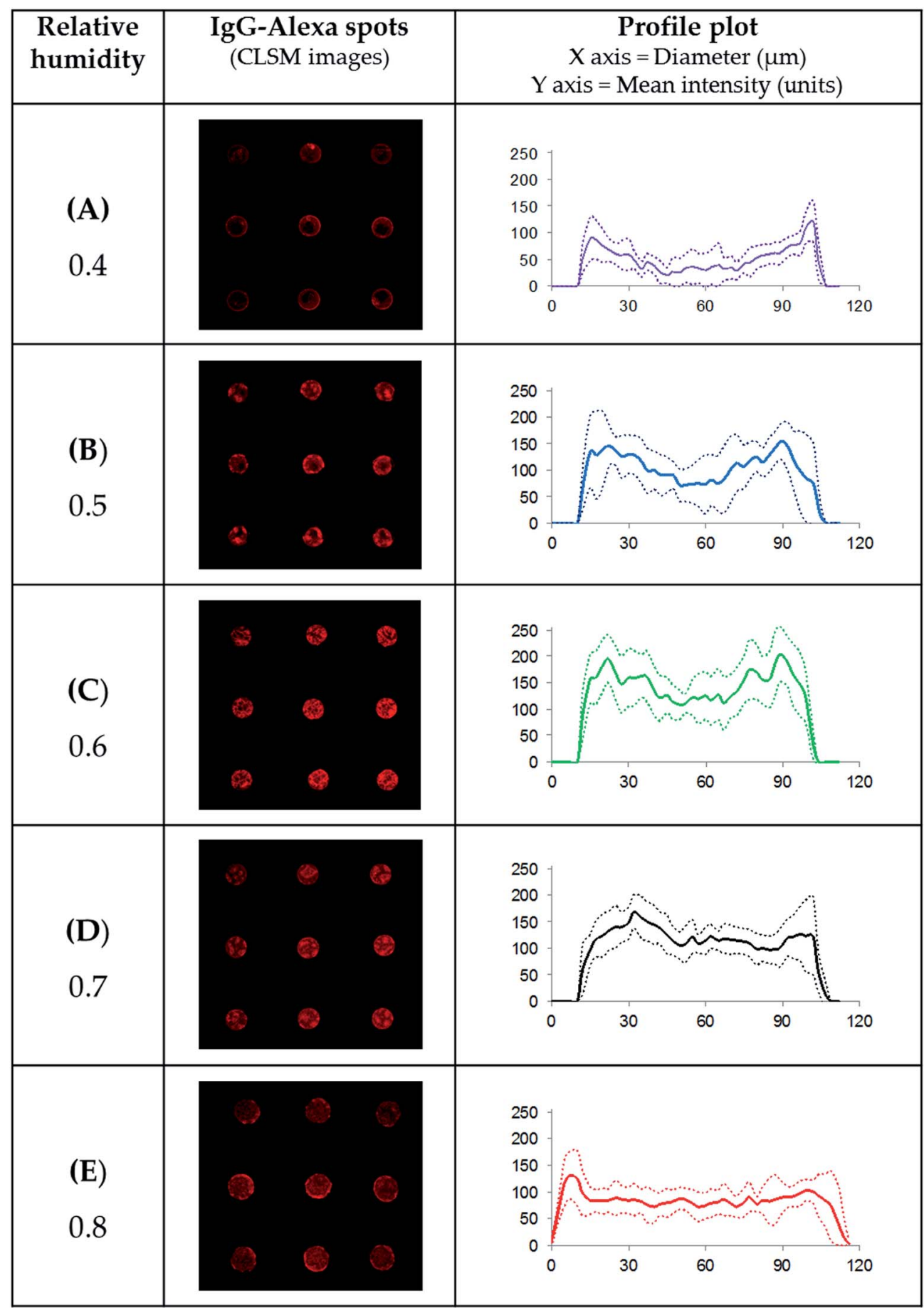

Fig. 6 Measured CLSM images of IgG-Alexa-635 printed and dried at (A) 40\%, (B) 50\%, (C) 60\%, (D) $70 \%$ and (E) $80 \pm 1 \%$ relative humidity. Also shown in the extreme right column is the intensity plot profile for these spots at the respective humidities along with the standard deviation.

increasing relative humidity, the distribution of the colloidal particles was more uniform. Similar studies on the relation between a pinned contact line and ring formation at lower humidity have been reported by Deegan et al. ${ }^{23}$

In our study, we focused on establishing optimal humidity conditions for obtaining a more homogeneous distribution of printed IgG molecules on the substrate HTA-PS slide. The experimental results were in line with theoretical simulations.
To overcome the coffee-stain effect, Eral et al. demonstrated the process of electrowetting. ${ }^{38}$ They showed that the applied electrostatic forces prevented the three phase contact line and generated an internal flow field, thereby preventing the accumulation of solutes along the contact line. Additionally, researchers have shown that the incorporation of additives to the printing buffer can also improve the non-homogeneous distribution in an inkjet printed spot. ${ }^{39-43}$ The influence of a 
polymeric additive to improve the functionality, i.e. the antigen binding capacity, of printed antibody molecules is the subject of our next paper.

\section{Conclusions}

Through numerical simulations and experiments, we have demonstrated the influence of the relative humidity on the mass distribution of inkjet-printed fluorophore labeled IgG molecules on non-porous substrates. It was found that at low humidity, the printed molecules are non-homogeneously distributed, thus resulting in coffee-stain-shaped spots. With increasing relative humidity, the coffee-stain-like appearance decreased and a more homogeneously distributed spot morphology was achieved. The best morphological results were obtained at a relative humidity of $70 \%$, but with respect to mean and total fluorescence intensities, we conclude that the optimum relative humidity for printing the IgG molecules onto non-porous substrates is between 60 and $70 \%$ relative humidity. The results compare favorably with the results of a numerical model in which it is assumed that the contact line of the droplet is pinned during the evaporation process.

\section{Acknowledgements}

This research was supported by the Dutch Technology Foundation STW, Applied-Science Division of NWO (Dutch Organisation for Scientific Research), and the Technology Program of the Ministry of Economic Affairs of The Netherlands.

\section{References}

1 J. N. Talbert, F. He, K. Seto, S. R. Nugen and J. M. Goddard, Enzyme Microb. Technol., 2014, 55, 21-25.

2 N. Komuro, S. Takaki, K. Suzuki and D. Citterio, Anal. Bioanal. Chem., 2013, 405, 5785-5805.

3 T. Soga, Y. Jimbo, K. Suzuki and D. Citterio, Anal. Chem., 2013, 85, 8973-8978.

4 W. Shen, M. Li, C. Ye, L. Jiang and Y. Song, Lab Chip, 2012, 12, 3089-3095.

5 X. Li, J. Tian, G. Garnier and W. Shen, Colloids Surf., B, 2010, 76, 564-570.

6 H. H. Lee, K. S. Chou and K. C. Huang, Nanotechnology, 2005, 16, 2436-2441.

7 P. Arenkov, A. Kukhtin, A. Gemmell, S. Voloshchuk, V. Chupeeva and A. Mirzabekov, Anal. Biochem., 2000, 278, 123-131.

8 S. Choudhuri, J. Biochem. Mol. Toxicol., 2004, 18, 171-179.

9 I. Barbulovic-Nad, M. Lucente, Y. Sun, M. Zhang, A. R. Wheeler and M. Bussmann, Crit. Rev. Biotechnol., 2006, 26, 237-259.

10 L. H. Mujawar, W. Norde and A. van Amerongen, Analyst, 2013, 138, 518-524.

11 R. L. DeRosa, J. A. Cardinale and A. Cooper, Thin Solid Films, 2007, 515, 4024-4031.

12 M. Dufva, Biomol. Eng., 2005, 22, 173-184.
13 W. Kusnezow and J. D. Hoheisel, J. Mol. Recognit., 2003, 16, 165-176.

14 Y. Liu, C. M. Li, W. Hu and Z. Lu, Talanta, 2009, 77, 11651171.

15 L. H. Mujawar, A. van Amerongen and W. Norde, Talanta, 2012, 98, 1-6.

16 L. H. Mujawar, A. Moers, W. Norde and A. Van Amerongen, Anal. Bioanal. Chem., 2013, 405, 7469-7476.

17 V. H. Chhasatia, A. S. Joshi and Y. Sun, Appl. Phys. Lett., 2010, 97, 231909.

18 C. Liu, E. Bonaccurso and H.-J. Butt, Phys. Chem. Chem. Phys., 2008, 10, 7150-7157.

19 M. J. Mackel, S. Sanchez and J. A. Kornfield, Langmuir, 2007, 23, 3-7.

20 W. Kusnezow, A. Jacob, A. Walijew, F. Diehl and J. D. Hoheisel, Proteomics, WILEY-VCH Verlag GmbH \& Co. KGaA, Weinheim, Germany, 2003, pp. 254-264.

21 R. D. Deegan, O. Bakajin, T. F. Dupont, G. Huber, S. R. Nagel and T. A. Witten, Nature, 1997, 389, 827-829.

22 R. D. Deegan, O. Bakajin, T. F. Dupont, G. Huber, S. R. Nagel and T. A. Witten, Phys. Rev. E: Stat. Phys., Plasmas, Fluids, Relat. Interdiscip. Top., 2000, 62, 756-765.

23 R. D. Deegan, Phys. Rev. E: Stat. Phys., Plasmas, Fluids, Relat. Interdiscip. Top., 2000, 61, 475-485.

24 A. P. Sommer and N. Rozlosnik, Cryst. Growth Des., 2005, 5, 551-557.

25 B. J. Fischer, Langmuir, 2002, 18, 60-67.

26 D. B. van Dam and J. G. M. Kuerten, Langmuir, 2008, 24, 582589.

27 S. Howison, Practical Applied Mathematics: Modelling, Analysis, Approximation, Cambridge University Press, UK, 2005, p. 1, ISBN 13 978-0-521-84274-3.

28 J. G. M. Kuerten and D. P. Siregar, Drying of inkjet-printed droplets, in Inkjet-based micromanufacturing, ed. J. G. Korvink, P. J. Smith and D.-Y. Shin, Wiley-VCH, 2012.

29 Y. O. Popov, Phys. Rev. E: Stat., Nonlinear, Soft Matter Phys., 2005, 71, 036313.

30 D. P. Siregar, J. G. Kuerten and C. W. van der Geld, J. Colloid Interface Sci., 2013, 392, 388-395.

31 R. Kurrat, J. J. Ramsden and J. E. Prenosil, J. Chem. Soc., Faraday Trans., 1994, 90, 587-590.

32 P. Van Dulm and W. Norde, J. Colloid Interface Sci., 1983, 91, 248-255.

33 C. Bourges-Monnier and M. E. R. Shanahan, Langmuir, 1995, 11, 2820-2829.

34 D. S. Golovko, H. J. Butt and E. Bonaccurso, Langmuir, 2009, 25, 75-78.

35 G. Guena, C. Poulard and A. M. Cazabat, Colloid J., 2007, 69, 1-8.

36 C. Lages and E. Mèndez, Anal. Bioanal. Chem., 2007, 388, 1689-1692.

37 C.-H. Choi and C.-J. C. Kim, Langmuir, 2009, 25, 75617567.

38 H. B. Eral, D. M. Augustine, M. H. G. Duits and F. Mugele, Soft Matter, 2011, 7, 4954-4958.

39 Y. Deng, X. Y. Zhu, T. Kienlen and A. Guo, J. Am. Chem. Soc., 2006, 128, 2768-2769. 
40 F. Diehl, S. Grahlmann, M. Beier and J. D. Hoheisel, Nucleic Acids Res., 2001, 29, E38.

41 M. K. McQuain, K. Seale, J. Peek, S. Levy and F. R. Haselton, Anal. Biochem., 2003, 320, 281-291.
42 K. Pappaert, H. Ottevaere, H. Thienpont, P. Van Hummelen and G. Desmet, BioTechniques, 2006, 41, 609-616.

43 P. Wu and D. W. Grainger, J. Proteome Res., 2006, 5, 29562965. 